\title{
Molecular Basis for Ultraviolet Vision in Invertebrates
}

\author{
Ernesto Salcedo, ${ }^{1}$ Lijun Zheng, ${ }^{1}$ Meridee Phistry, ${ }^{1}$ Eve E. Bagg, ${ }^{1}$ and Steven G. Britt ${ }^{1,2}$ \\ Departments of ${ }^{1}$ Cell and Developmental Biology, and ${ }^{2}$ Ophthalmology, University of Colorado Health Sciences Center, Denver, Colorado 80262
}

Invertebrates are sensitive to a broad spectrum of light that ranges from UV to red. Color sensitivity in the UV plays an important role in foraging, navigation, and mate selection in both flying and terrestrial invertebrate animals. Here, we show that a single amino acid polymorphism is responsible for invertebrate UV vision. This residue (UV: lysine vs blue:asparagine or glutamate) corresponds to amino acid position glycine 90 (G90) in bovine rhodopsin, a site affected in autosomal dominant human congenital night blindness. Introduction of the positively charged lysine in invertebrates is likely to deprotonate the Schiff base chromophore and produce an UV visual pigment. This same position is responsible for regulating UV versus blue sensitivity in several bird species, suggesting that UV vision has arisen independently in invertebrate and vertebrate lineages by a similar molecular mechanism.

Key words: Drosophila; ERG (electroretinogram); photoreceptor; retina; vision; rhodopsin

\section{Introduction}

Color vision is one of the most familiar forms of stimulus discrimination and is dependent on an organism having at least two different classes of photoreceptor cells of different spectral sensitivities (SS) (Jacobs, 1981). Except in eyes using screening pigments or oil droplets, this is based on the expression of spectrally distinct forms of the visual pigment rhodopsin, in different photoreceptor cells. Invertebrates are sensitive to a broad spectrum of light that ranges from UV to red in some species (Gärtner, 2000; Briscoe and Chittka, 2001). UV vision is used in foraging, navigation, and mate selection in both flying and terrestrial invertebrate animals (Tovee, 1995). UV vision is mediated by a form of rhodopsin, the visual pigment of both vertebrate and invertebrate organisms. Rhodopsin is composed of an 11-cis-retinal chromophore that is attached covalently through a Schiff base linkage to a lysine residue in the opsin apoprotein (Fig. 1a). The absorption spectrum of the visual pigment determines the SS of the photoreceptor in which it is expressed, and this in turn is dependent on specific interactions between the opsin apoprotein and the retinal chromophore. Drosophila melanogaster uses six visual pigments that range in absorption from UV to green (Fig. 1b) (Salcedo et al., 1999). These diverse pigments provide an opportunity to examine the basis for spectral tuning in insects and other invertebrates. Furthermore, transgenic flies can be used as an expression system to examine the function of novel or modified visual pigments in vivo (Feiler et al., 1988, 1992; Britt et al., 1993; Townson et al., 1998; Salcedo et al., 1999).

Previous studies of bovine rhodopsin have shown that a chromophore/apoprotein interaction occurs between the protonated

\footnotetext{
Received Sept. 3, 2003; revised Sept. 30, 2003; accepted Oct. 1, 2003.

This work was supported by National Institutes of Health-National Eye Institute Grant R01-EY10759 (S.G.B.). We thank L. Chadwell, S. Li, W. Yu, and J. Hoppin for assistance, as well as J. Hooper and T. Finger for comments on this manuscript.

Correspondence should be addressed to Dr. Steven G. Britt, Departments of Cell and Developmental Biology and Ophthalmology, University of Colorado Health Sciences Center, 4200 East Ninth Avenue, B-111, Denver, C0 80262. E-mail: steve.britt@uchsc.edu.

Copyright $\odot 2003$ Society for Neuroscience $\quad$ 0270-6474/03/2310873-06\$15.00/0
}

Schiff base nitrogen and a negatively charged counter-ion at position glutamate 113 (E113) (Fig. 1a) (Sakmar et al., 1989; Zhukovsky and Oprian, 1989; Nathans, 1990). This interaction stabilizes the protonated Schiff base and is responsible for the opsin red shift that converts both UV absorbing chromophore and apoprotein into a visible absorbing complex. Removal of the negative charge of the counter-ion results in deprotonation of the chromophore and yields a UV-absorbing pigment. In invertebrate visual pigments, the position homologous to bovine rhodopsin E113 is occupied by a tyrosine $(\mathrm{Y})$ or phenylalanine $(\mathrm{F})$ residue in the visible and UV-absorbing invertebrate pigments, respectively (Fig. 2). This site has been proposed as the invertebrate counterion, and the F/Y polymorphism may be responsible for the difference in absorption between the visible and UV pigments of invertebrates.

In the present study, we performed a series of experiments to test the hypothesis that the F/Y polymorphism is responsible for the difference in absorption between UV and visible absorbing pigments. We found that this polymorphism is not responsible for UV sensitivity and then conducted a comparative phylogenetic analysis of a group of closely related visible and UV-absorbing invertebrate rhodopsins in an effort to identify additional candidate residues. We show that one of these polymorphisms is responsible for UV vision in invertebrates and present both a model of the underlying mechanism of wavelength regulation and a test of the model that indicates the effect of the polymorphism is to deprotonate the Schiff base chromophore and produce a UV visual pigment.

\section{Materials and Methods}

Ectopic expression of native and modified Drosophila opsin genes. Flies expressing the $R h 3$ and $R h 5$ opsin genes in the R1-6 photoreceptor cells of ninaE mutants have been described previously (Feiler et al., 1992; Chou et al., 1996; Salcedo et al., 1999). Flies expressing modified forms of $R h 1, R h 3$, and $R h 5$ were generated in a similar manner. Briefly, the gene encoding the opsin of interest was modified by site-directed mutagenesis using inverse PCR with Pfu DNA polymerase and DpnI digestion of the methylated template (Weiner et al., 1994; Fisher and Pei, 1997). The sequence of the mutagenized fragment was confirmed, and the fragment 
a

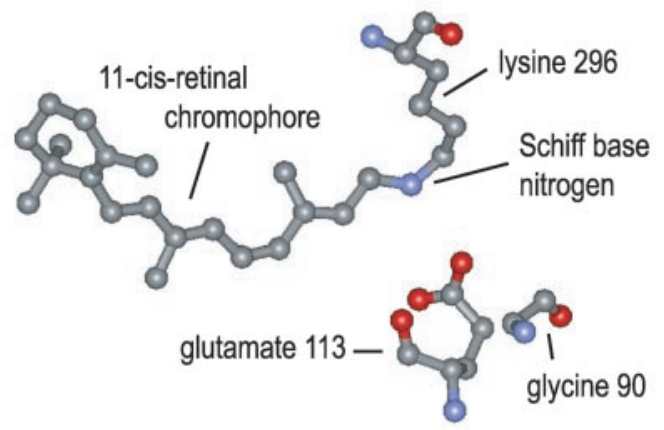

b
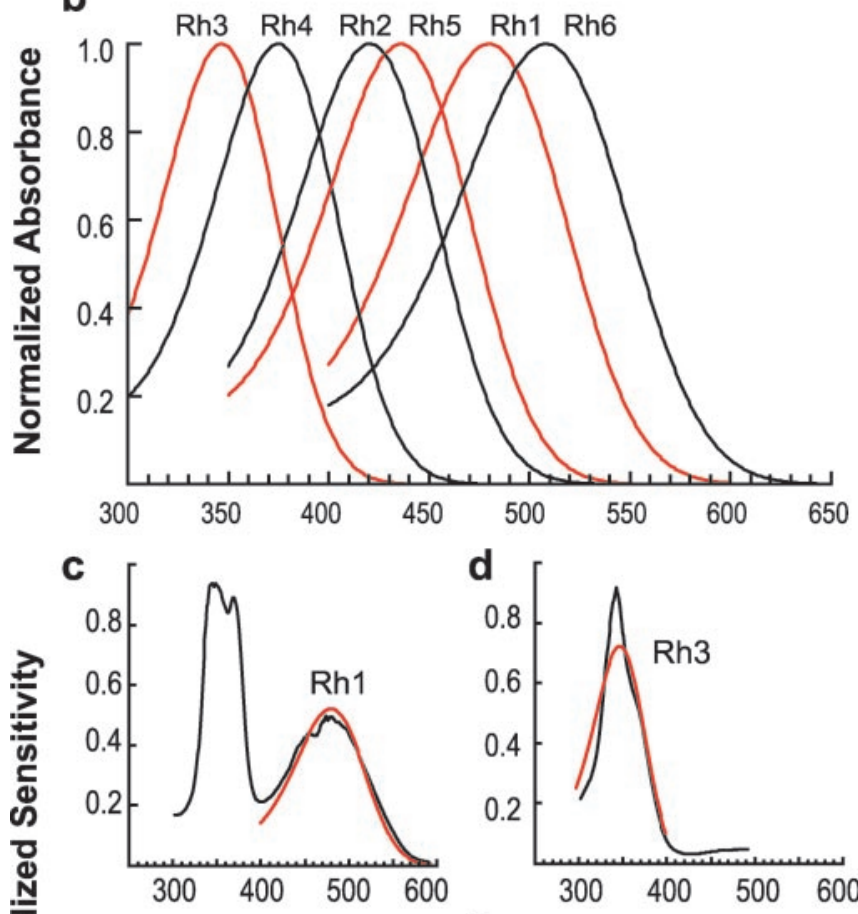

d

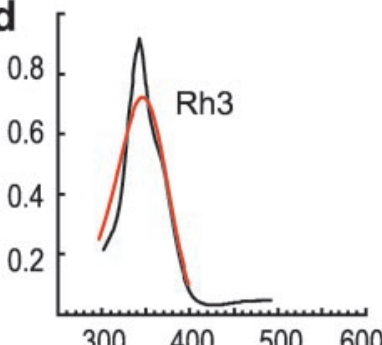

e

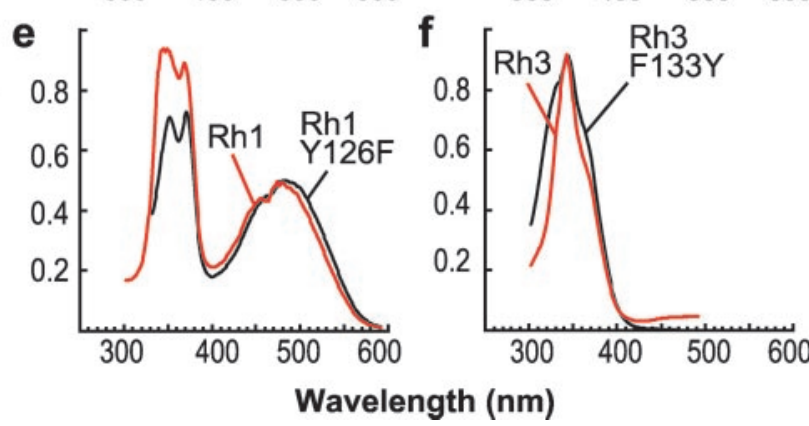

Figure 1. Chromophore structure, rhodopsin absorption, and spectral sensitivity. a, 11-cisRetinal chromophore attached to lysine 296 as the Schiff base with the counter-ion (glutamate 113 ) and glycine 90 are shown, from the bovine rhodopsin crystal structure (Palczewski et al., 2000). Gray, Carbon; blue, nitrogen; red, oxygen. $b$, Calculated absorption profiles of Drosophila rhodopsins $\operatorname{Rh} 1\left(\lambda_{\max }, 480 \mathrm{~nm}\right), \operatorname{Rh} 2\left(\lambda_{\max }, 420 \mathrm{~nm}\right)$ (Feiler et al., 1988), $\operatorname{Rh} 3\left(\lambda_{\max }, 347 \mathrm{~nm}\right)$, $\operatorname{Rh} 4\left(\lambda_{\max }, 375 \mathrm{~nm}\right)$ (Feiler et al., 1992$), \operatorname{Rh} 5\left(\lambda_{\max }, 436 \mathrm{~nm}\right)$, and Rh6 $\left(\lambda_{\max }, 508 \mathrm{~nm}\right)$ (Salcedo et al., 1999). Rh1, Rh3, and Rh5 are shown in red. c, d, Measured SS of flies expressing $R h 1$ (c) and $R h 3(d)$ in the R1-6 photoreceptor cells. A curve fit of the rhodopsin nomogram is shown in red. Flies expressing $R h 1$ (c) in the R1- 6 photoreceptor cells display a dual peak of sensitivity. The peak in the UV region occurs because of the action of a sensitizing pigment that absorbs in the UV and activates the Rh1 rhodopsin through energy transfer (Kirschfeld et al., 1977). The peak in the visible region (with red curve fit) is attributable to direct absorption by Rh1. $e$, $f$, SS of flies expressing rhodopsin mutants (e, Rh1Y126F; $f$, Rh3 F133Y) compared with flies expressing the unmodified pigments (red traces). Each sensitivity spectrum obtained in this study was fit to a rhodopsin absorption nomogram. The $\lambda_{\text {max }}$, correlation coefficient, and number of flies examined are shown in Table 1. was then subcloned into an expression cassette containing the $R h 1$ promoter (2.4 $\mathrm{kb}$ including $33 \mathrm{bp}$ of the $5^{\prime}$ untranslated region) and the remainder of the opsin gene of interest. The transcriptional fusion encoding modified $R h 3, R h 5$, or the modified form of $R h 1$ was subcloned into the $y^{+}$-marked P-element vector "C4" obtained from Pam Geyer (University of Iowa, Iowa City, IA) (Patton et al., 1992). The construct was injected into $y w$; sr ninaE ${ }^{17}$ mutant embryos, and multiple independent P-element-mediated germ line transformants were obtained (Karess and Rubin, 1984).

Electrophysiology. Electroretinogram (ERG) recordings were obtained from immobilized white-eyed $(w)$ flies using glass microelectrodes filled with normal saline $(0.9 \% \mathrm{NaCl}, \mathrm{w} / \mathrm{v})$ (Salcedo et al., 1999). Flies were stimulated with light from a xenon arc lamp (350 W; Osram; Oriel, Stratford, CT), using interference and neutral density filters. Light intensity was measured using a calibrated silicon photodiode (model 71883; Oriel) and an optical power meter (model 70310; Oriel).

SS was measured using a modification of the voltage-clamp method of Franceschini $(1979,1984)$, which we have described in detail previously (Townson et al., 1998; Salcedo et al., 1999). Briefly, the amplitude of the ERG response to a flickering $(10 \mathrm{~Hz})$ monochromatic stimulus was maintained at a criterion level by adjusting the light intensity, whereas the wavelength of stimulating light was varied during a scan. In the measurements in this study, for each $\sim 0.3 \mathrm{sec}$ window (ERG response to approximately three flickers), we averaged all of the ERG voltages during this period and used the average deviation as an estimate of the amplitude (width) of the response (Press et al., 1992). The average deviation is a function of the variance between the individual data points and the mean voltage during the sinusoidal ERG response. Thus, the average deviation is related to the response amplitude, although much less sensitive to baseline drift and noise.

During an experiment, as the monochrometer was stepped through a scan in $0.1 \mathrm{~nm}$ increments, the average deviation of the ERG response was calculated and compared with a criterion set point. The ERG response was maintained near the set point during the scan by constantly adjusting the light intensity using a proportional-integral-derivative algorithm (Corripio, 1990). SS was defined as the inverse of the light flux required to produce the criterion response, taking into account the wavelength and intensity of the stimulating light [i.e., SS $\propto 1 /$ (light intensity $(\mu \mathrm{W} /$ $\mathrm{cm}^{2}$ ) $\times$ wavelength)]. Raw sensitivity data were normalized to an amplitude of 1.0 at the wavelength of maximum sensitivity, multiple individual measurements were averaged, and values that differed by $>10 \%$ from the mean value within a $10 \mathrm{~nm}$ window were filtered out. The filtered spectra were then smoothed with a window of $10 \mathrm{~nm}$.

Experiments were performed on a minimum of four individual flies, two each from two different transgenic lines for each construct. We typically record from many more animals and lines as well, although these may not all have been included in the calculations. Occasional discrepancies occur between recordings that we have attributed to individual differences (health, age, light exposure, etc.). We have never observed any instances in which one transgenic line with a particular construct was consistently different from another. The sampling interval of the instrument was $\sim 0.2 \mathrm{~nm}$, taking into account the scan rate of the monochrometer $(0.5 \mathrm{~nm} / \mathrm{sec})$ and the sampling rate for determining the response amplitude (three per second). The monochromator was calibrated using an Oriel 6025 mercury (argon) calibration lamp with spectral lines at $312.6,365.0,404.7,435.8,546.7,577.0$, and $579.1 \mathrm{~nm}$. Monochromator error was within the level of resolution of the instrument, over the wavelength range from 300 to $600 \mathrm{~nm}( \pm 1.0 \mathrm{~nm}$ at a slit width of 280 $\mu \mathrm{m}$ ). Curve fitting of spectral sensitivity recordings to rhodopsin nomograms (see below) was within $\pm 1.0 \mathrm{~nm}$. Variation between individual measurements was typically within $1-2 \mathrm{~nm}$.

ERG and SS recordings were performed on transgenic animals expressing native or modified forms of rhodopsin in either a nina $E^{17}$ background or in a modified norpA; nina $E^{17}$ mutant background. The latter strain also contained an additional transgene driving the norpA cDNA in the R1-6 photoreceptor cells under the control of the Rh1 promoter. This background strain allows the activity of the modified pigment to be examined without interference from the R7 and R8 cells that are not affected by the ninaE mutation (Salcedo et al., 1999). 


Carpenter Ant UV
Desert Ant UV
Bee UV

Figure 2. Phylogenetic and amino acid comparison of UV- and blue-absorbing invertebrate visual pigments. The phylogenetic tree is derived from Salcedo et al. (1999). Amino acids modified in the mutagenesis study are indicated by symbol and position, and the two pigments that were modified ( $R h 3$ and $R h 5$ ) are indicated by asterisks. The amino acid polymorphism responsible for insect UV vision in the $R h 3$ and $R h 5$ sequences is boxed in contrast. Bovine rhodopsin is shown for comparison.

Rhodopsin nomogram modeling. Rhodopsin absorption spectra were calculated from the SS recordings using the exponential function described by Stavenga et al. (1993). Briefly, the spectral shape of the rhodopsin $\alpha$-band absorption can be described by the following lognormal function:

$$
\alpha=A \exp \left[-a_{0} x^{2}\left(1+a_{1} x+a_{2} x^{2}\right)\right]
$$

where $x={ }^{10} \log \left(\lambda / \lambda_{\max }\right), A=1, a_{0}=380, a_{1}=6.09$ and $a_{2}=3 a_{1}{ }^{2} / 8$.

A curve-fitting routine was implemented in KaleidaGraph (version 3.51; Synergy Software, Reading, PA) using the Levenberg-Marquardt (nonlinear least-squares) algorithm (Press et al., 1992). The computer solved for the $\lambda_{\max }$ and amplitude of the rhodopsin absorption spectra and calculated the SD for each variable and the correlation coefficient (Pearson's $r$ ).

Each sensitivity spectrum obtained in this study was fit to a rhodopsin absorption nomogram. The $\lambda_{\max }$, correlation coefficient, and number of flies examined are indicated in Table 1.

\section{Results}

In our first series of experiments, we tested the hypothesis that the F/Y polymorphism between UV and visible absorbing pigments, which occurs at the same position as the vertebrate counter-ion (E113), is responsible for UV vision. We constructed mutant forms of a blue-absorbing (Rh1) and UV-absorbing (Rh3) Drosophila rhodopsin in which the residue at this position was replaced with that found in the other class of pigments (i.e., Rh1 Y126F and Rh3 F133Y). To determine the effect of the amino acid change on the absorption of the visual pigment, the genes encoding these modified pigments were introduced into the germ line of transgenic Drosophila containing the ninaE mutation, which is a deletion in the endogenous $R h 1$ gene that is expressed in the R1-6 photoreceptor cells (O'Tousa et al., 1985; Zuker et al., 1985). We found that the SS of transgenic animals expressing either Rh1 Y126F or Rh3 F133Y showed very little change from that of the unmodified pigments (Fig. $1 c-f$ ). These results indicate that the F/Y polymorphism at the position of bovine E113 is not responsible for the difference in SS between the invertebrate $\mathrm{UV}$ and visible absorbing pigments and suggests that the invertebrate counter-ion is located elsewhere. These results are also consistent with the finding that the invertebrate tyrosine is not ionized and is, thus, unlikely to serve as a true counter-ion to the protonated Schiff base (Hashimoto et al., 1996; Nakagawa et al., 1999).

To identify additional candidate residues responsible for UV versus visible sensitivity, we undertook a phylogenetic analysis of a group of well characterized invertebrate visual pigments. Figure 2 shows that a group of blue-absorbing visual pigments (e.g., Rh5) are closely related to, and seem to share a common ancestor with, the UV-absorbing pigments of invertebrates (e.g., Rh3) (Salcedo et al., 1999). This suggests that during evolution one or both of these groups acquired amino acid changes that are responsible for the differences in their spectral properties. Figure 2 shows a group of polymorphic amino acids that are conserved within each group (UV or visible absorbing) but differ between them and might be expected to affect chromophore absorption based on side chain position and differences in polarity or charge. Nine modified forms of the gene encoding Rh5 (blue absorbing) containing these 12 amino acid changes, as single or double amino acid substitutions, were introduced into ninaE mutant flies. Electrophysiological analysis of the animals expressing the transgenes showed little or no change in SS for virtually all of the $R h 5 \mathrm{mu}$ tants. One modified pigment, Rh5 N104K, showed a dramatic shift in SS (from $\lambda_{\max }=436-377 \mathrm{~nm}$ ) (Fig. 3a). To confirm that the lysine $(\mathrm{K})$ versus asparagine $(\mathrm{N})$ polymorphism is responsible for a large component of the difference between UV and visible absorbing pigments in invertebrates, we replaced the existing lysine residue in $\mathrm{Rh} 3$ with the corresponding amino acid found in Rh5. Transgenic flies expressing the modified Rh3 K110N opsin showed a complementary shift in SS (from $\lambda_{\max }=347-420 \mathrm{~nm}$ ) (Fig. $3 b$ ). This confirms that the $\mathrm{K}$ versus $\mathrm{N}$ polymorphism is responsible for UV sensitivity.

The blue-absorbing pigments have either an asparagine (in the case of Rh5) or a glutamate (E) (in the case of the Bee blue and other members of this class) at this position (Fig. 2). To test whether the asparagine (neutral) versus glutamate (negatively charged) polymorphism may also affect spectral tuning, we generated the Rh3 K110E mutant and found that its SS is identical to Rh3 K110N (Fig. 3b). This indicates that replacement of the lysine with a neutral (asparagine) or negatively charged (glutamate) residue has the same effect, suggesting it is the removal of the positively charged lysine that converts Rh3 to a visible absorbing pigment. Because the mutant pigments are not fully shifted to the $\lambda_{\max }$ of the related pigment, these experiments also demonstrate that additional amino acid polymorphisms must contribute to differences in SS. This is expected given that the Drosophila UV-absorbing and visible absorbing pigments differ considerably among themselves (e.g., the $\lambda_{\max }$ of UV pigments Rh3 and Rh4 differ by $28 \mathrm{~nm}$ ) (Fig. $1 b$ ).

The position within bovine rhodopsin corresponding to this site is glycine 90 (G90), which has been associated with the human congenital night blindness mutation G90D (Rao et al., 1994). In addition, a cysteine/serine (C/S) polymorphism at this site is responsible for $\sim 35 \mathrm{~nm}$ difference in absorption between the UV and blue-absorbing visual pigments of birds, although the precise molecular mechanism underlying this effect is not known (Wilkie et al., 2000; Yokoyama et al., 2000). An analysis of struc- 
Table 1. $\boldsymbol{\lambda}_{\max }$ and rhodopsin absorption nomogram curve fit data for each wildtype and mutant pigment examined in the study

\begin{tabular}{|c|c|c|c|c|}
\hline Visual pigment & $\lambda_{\max }$ & $\begin{array}{l}\text { Correlation } \\
\text { coefficient of } \\
\text { curve fit }\end{array}$ & $\begin{array}{l}\text { Number of } \\
\text { flies analyzed }\end{array}$ & Figure \\
\hline Rh1 & $480 \mathrm{~nm}$ & 0.981 & 3 & $1 c, 4 a$ \\
\hline Rh3 & $347 \mathrm{~nm}$ & 0.969 & 11 & $1 d, 3 b, 4 b$ \\
\hline Rh1 Y126F & $486 \mathrm{~nm}$ & 0.988 & 8 & $1 e$ \\
\hline Rh3 F133Y & $344 \mathrm{~nm}$ & 0.994 & 11 & $1 f$ \\
\hline Rh5 & $436 \mathrm{~nm}$ & 0.997 & 8 & $3 a$ \\
\hline Rh5 H38P, R42L & $434 \mathrm{~nm}$ & 0.994 & 4 & $3 a$ \\
\hline Rh5 A53Y & $435 \mathrm{~nm}$ & 0.995 & 4 & $3 a$ \\
\hline Rh5 N104K & $377 \mathrm{~nm}$ & 0.990 & 5 & $3 a$ \\
\hline Rh5 I110Y & $434 \mathrm{~nm}$ & 0.999 & 4 & $3 a$ \\
\hline Rh5 D125Q, Y127F & $436 \mathrm{~nm}$ & 0.996 & 4 & $3 a$ \\
\hline Rh5 F147Y & $437 \mathrm{~nm}$ & 0.994 & 3 & $3 a$ \\
\hline Rh5 T173I, W176Y & $434 \mathrm{~nm}$ & 0.996 & 3 & $3 a$ \\
\hline Rh5 R243F & $435 \mathrm{~nm}$ & 0.996 & 3 & $3 a$ \\
\hline Rh5 S325A & $437 \mathrm{~nm}$ & 0.995 & 3 & $3 a$ \\
\hline Rh3 K110N & $420 \mathrm{~nm}$ & 0.970 & 10 & $3 b$ \\
\hline Rh3 K110E & $421 \mathrm{~nm}$ & 0.943 & 8 & $3 b$ \\
\hline Rh1 Y126E & $488 \mathrm{~nm}$ & 0.994 & 7 & $4 a$ \\
\hline Rh3 F133E & $371 \mathrm{~nm}^{1}$ & 0.246 & 9 & $4 b, c$ \\
\hline Rh3 F133E & $\begin{array}{l}345 \mathrm{~nm} \\
420 \mathrm{~nm}^{2}\end{array}$ & 0.988 & 9 & $4 b, d$ \\
\hline
\end{tabular}

'Single pigment fit.

${ }^{2}$ Two pigment fit; ratio of pigments 1:1.74

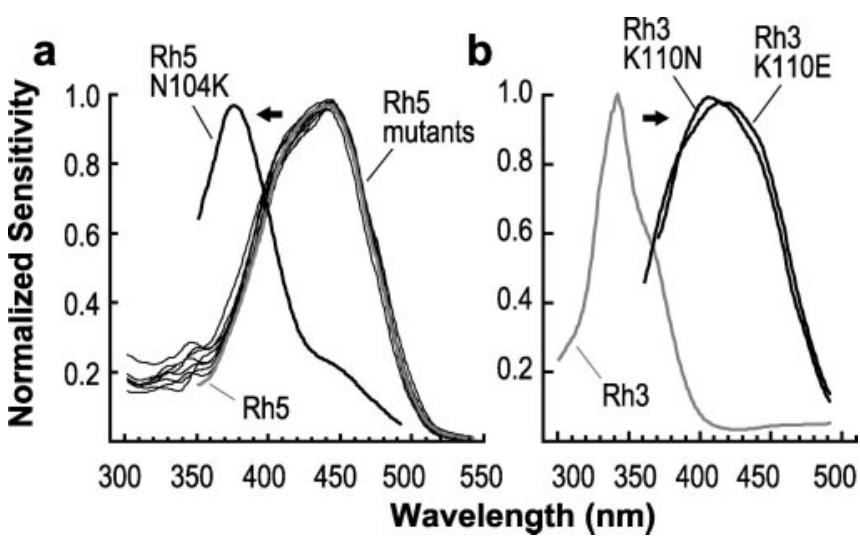

Figure 3. SS of flies expressing mutant Drosophila $R h 5$ and $R h 3$ rhodopsins. $a, A$ single $R h 5$ mutant shows a dramatic shift into the UV (Rh5 N104K). $b$, Reciprocal mutations in Rh3 show a dramatic shift into the visible region (Rh3 K110N and Rh3 K110E). Flies expressing the unmodified pigments are labeled and indicated as gray lines.

tural models of these pigments based on the bovine rhodopsin crystal structure (Palczewski et al., 2000) has suggested that, in the UV pigments, the thiol group of $\mathrm{C} 90$ is positioned very close to E113 and may exist as a thiolate ion (Hunt et al., 2001). The authors propose that this may serve to further stabilize the proton on the Schiff base in the ground state, with a resulting shift of absorption into the UV. Alternatively, it has also been suggested that the cysteine residue may remove a water molecule from the region near the Schiff base and displace its positive charge. Deprotonation would be expected to produce a large shift into the UV region as well (Yokoyama et al., 2000).

Our finding that the introduction of a lysine residue at this position is sufficient to shift the absorption spectrum into the UV can be more easily interpreted. Comparative modeling of the helices of the Rh5 and Rh3 visual pigments reveals a similar geometry for the position of the $\mathrm{N} / \mathrm{K}$ polymorphism with respect to the Schiff base nitrogen, as that of G90 in bovine rhodopsin (data
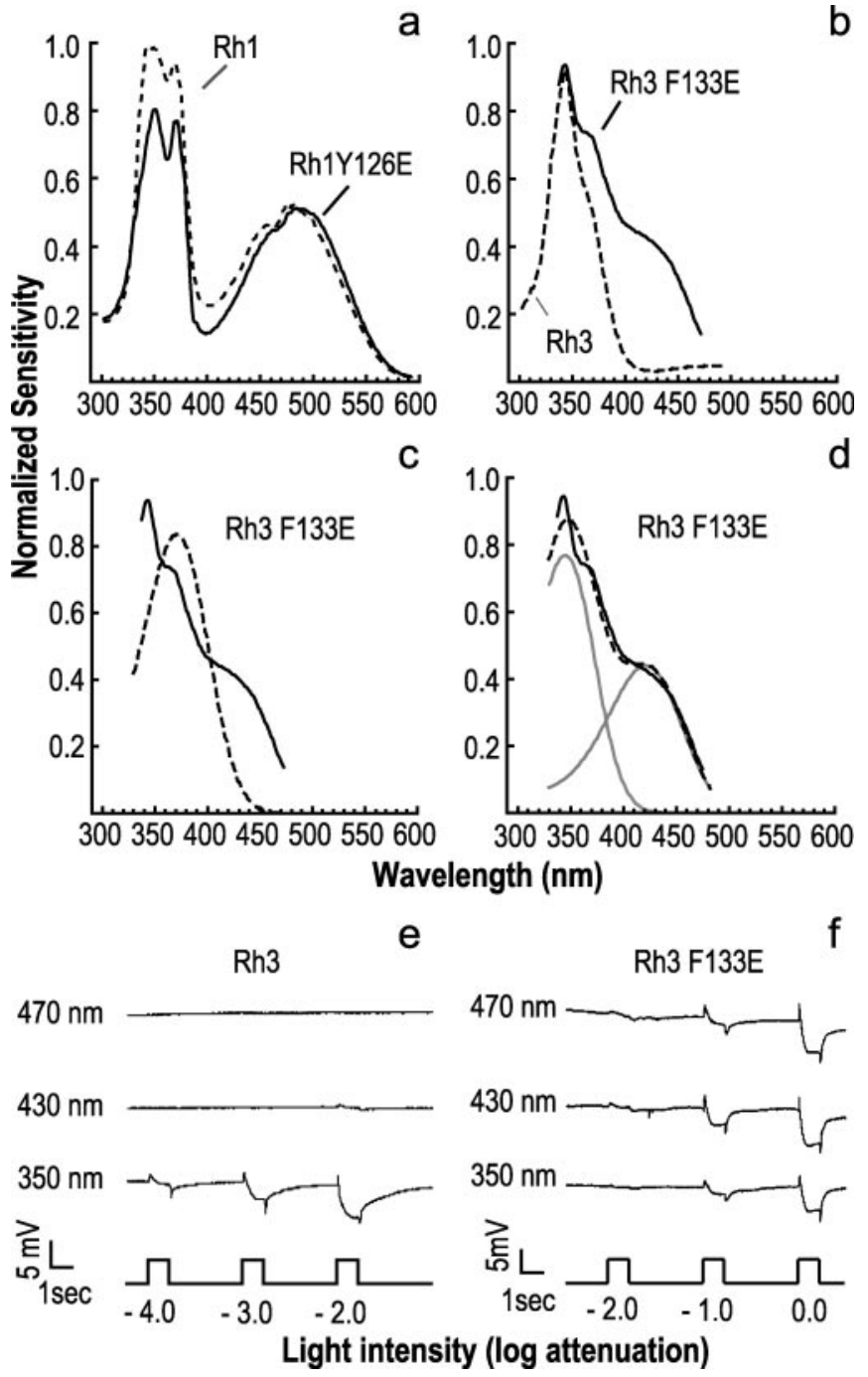

Figure 4. The effect of a negatively charged residue substituted into the region of the chromophore. Spectral sensitivity of flies expressing Rh1 Y126E ( $a$ ) and Rh3 F133E (b) with the unmodified pigments shown as broken lines. The sensitivity spectrum of Rh $3 \mathrm{~F} 133 \mathrm{E}$ is poorly fit by a single pigment absorption ( $c$, broken line) but well fit by two pigments ( $d$, sum trace broken line overlayed on measured curve; pigment pair shown as gray lines). Compared with flies expressing the unmodified Rh3 (e), ERG recordings confirm that flies expressing Rh3 F133E ( $f$ ) have additional sensitivity in the visible region. Wavelengths and light intensity (log attenuation) are shown.

not shown). Although the identity of the invertebrate counterion is not known, insertion of a positively charged lysine into the region of the positively charged Schiff base and negatively charged counter-ion would be expected to disrupt the interaction between them and lead to the deprotonation of the Schiff base. Although the phenylalanine/tyrosine at the position of the vertebrate counter-ion (E113) is apparently not the invertebrate counter-ion, this residue is likely to be in close proximity to the Schiff base in the pigments. If this is the case, one would predict that introduction of an additional negative charge at this site would block the affect of the lysine (at G90) in the UV pigment.

To test this hypothesis, we substituted a glutamic acid at the position of E113 in bovine rhodopsin into both Rh1 (in place of the native tyrosine Rh1 Y126E) as well as into Rh3 (in place of the native phenylalanine Rh3 F133E). Whereas introduction of this additional negative charge in Rh1 had only a small affect (Fig. 4a), its introduction into the UV pigment Rh3 (Fig. 4b) caused signif- 
icant broadening in the sensitivity spectrum. This change was best fit by absorption nomograms corresponding to a mixture of two (UV and blue absorbing) pigments (Fig. $4 c, d$ ). An analysis of ERG responses to flashes at varying wavelengths and intensities also confirmed that the Rh3 F133E mutant has a significant increase in sensitivity in the visible region $(430-470 \mathrm{~nm}$ ) (Fig. 4e,f). Thus, the introduction of an additional negative charge was sufficient to shift the equilibrium in the protonation of the Schiff base and produce a mixture of visual pigment states. Substitution with the glutamate residue may, thus, serve as an intramolecular titration that partially stabilizes the protonated Schiff base. Alternatively, the mutant pigment may adopt two different conformations in which the Schiff base is protonated in one and not in the other. In either case, these findings are consistent with the model that UV absorption in the invertebrate pigments is based on the deprotonation of the Schiff base by the introduction of a positively charged lysine residue. We believe a similar deprotonation event is responsible for the absorption of the avian UV pigments.

\section{Discussion}

The principal result from this study is that the $\mathrm{K}$ (versus $\mathrm{N}$ or $\mathrm{E}$ ) polymorphism present at the position of G90 in bovine rhodopsin is the basis for UV vision in invertebrates. The introduction of the lysine residue at this position seems to deprotonate the Schiff base and shift the absorption of the pigment into the UV. This deprotonation seems to change during photoactivation, in which photon absorption by rhodopsin leads to the isomerization of the 11-cis-retinal chromphore to the all-trans configuration and induces a conformational change in the apoprotein, leading to the formation of the activated form of the visual pigment metarhodopsin. Photoactivation of the Drosophila Rh3 and Rh4 UVabsorbing rhodopsins leads to the formation of metarhodopsin species that absorb in the blue region (Feiler et al., 1992; Salcedo et al., 1999). This likely results from the protonation of the Schiff base in the metarhodopsin form of the pigment (Pande et al., 1987) as is thought to be the case for the mouse UV pigment (Dukkipati et al., 2002). This would require a conformational change that either removes the lysine (at G90), introduces an additional negative charge into the region near the chromophore, or uses an alternate counter-ion site in metarhodopsin, as is thought to occur in the vertebrate visual pigments (Yan et al., 2003).

The G90 amino acid position has been the subject of extensive study in the vertebrate visual pigments. A C/S polymorphism at this position is responsible for UV vision in birds (Wilkie et al., 2000; Yokoyama et al., 2000), and amino acid substitutions at this site have also been shown to alter the absorption maxima of other visual pigments (Lin et al., 1998; Dukkipati et al., 2001; Janz and Farrens, 2001). Furthermore, this position is associated with the human congenital night blindness mutation G90D (Rao et al., 1994). The introduction of an aspartic acid residue has been shown to alter the absorption maximum of the pigment and to generate a constitutively activated opsin apoprotein (Rao et al., 1994). Consistent with this observation, expression of the mutant visual pigment in vivo reduces light sensitivity in a manner similar to light adaptation that can be suppressed with the addition of exogenous chromophore (Sieving et al., 2001; Jin et al., 2003).

The rhodopsin crystal structure shows that G90 is in close proximity to the protonated Schiff base and the counter-ion at E113 (Fig. 1a). In the G90D mutant, interactions between the protonated Schiff base and the counterion (E113) are altered. G90D seems to compete with E113 as the counter-ion for the protonated Schiff base, leaving E113 protonated in both the dark state and in subsequent photoproducts (Fahmy et al., 1996; Gross et al., 2003b). In addition, both the absorption maxima as well as the formation and decay kinetics of the mutant pigment photoproducts are altered (Zvyaga et al., 1996; Jager et al., 1997). Finally, the mutant opsin protein binds retinal more slowly than the wild-type protein, suggesting that the introduced aspartate may interfere with the normal catalysis of Schiff base formation (Gross et al., 2003a).

These findings highlight the close proximity of the G90 position to the chromophore and the degree to which an amino acid substitution at this site may alter chromophore-protein interactions and visual pigment function. From this perspective, it is interesting to note that some native invertebrate visual pigments contain a negatively charged residue at this position (e.g., Bee blue and others) (Fig. 2), suggesting that some aspects of the chromophore-binding site are different in invertebrates. This also indicates that the presence of a negative charge is not precluded from this location and suggests that there must be additional amino acid differences between vertebrate and invertebrate pigments that function to accommodate a negative charge in this position.

Although a C/S polymorphism at G90 has been shown to be the basis for UV vision in birds, polymorphisms at other sites are responsible for UV vision in fish, reptiles, and rodents (Shi et al., 2001; Cowing et al., 2002; Fasick et al., 2002). Phylogenetically, the ancestral vertebrate visual pigment was likely UV absorbing (like those from fish and rodents). The visible pigments are thought to have arisen independently in multiple clades, and the avian UV pigments are thought to be derived from a visible absorbing ancestor (having acquired the $\mathrm{C} / \mathrm{S}$ polymorphism) (Shi et al., 2001; Cowing et al., 2002). In contrast, all of the invertebrate visual pigments are thought to have arisen from a hypothetical ancestral pigment that was blue-green absorbing (Briscoe and Chittka, 2001). It is remarkable to note that although the polymorphisms responsible for UV vision in birds and insects seem to have occurred independently, they are at an identical site in the protein and likely based on a similar molecular mechanism.

\section{References}

Briscoe AD, Chittka L (2001) The evolution of color vision in insects. Ann Rev Entomol 46:471-510.

Britt SG, Feiler R, Kirschfeld K, Zuker CS (1993) Spectral tuning of rhodopsin and metarhodopsin in vivo. Neuron 11:29-39.

Chou WH, Hall KJ, Wilson DB, Wideman CL, Townson SM, Chadwell LV, Britt SG (1996) Identification of a novel Drosophila opsin reveals specific patterning of the R7 and R8 photoreceptor cells. Neuron 17:1101-1115.

Corripio AB (1990) Tuning of industrial control systems. Research Triangle Park, NC: Instrument Society of America.

Cowing JA, Poopalasundaram S, Wilkie SE, Bowmaker JK, Hunt DM (2002) Spectral tuning and evolution of short wave-sensitive cone pigments in cottoid fish from Lake Baikal. Biochemistry 41:6019-6025.

Dukkipati A, Vought BW, Singh D, Birge RR, Knox BE (2001) Serine 85 in transmembrane helix 2 of short-wavelength visual pigments interacts with the retinylidene Schiff base counterion. Biochemistry 40:15098-15108.

Dukkipati A, Kusnetzow A, Babu KR, Ramos L, Singh D, Knox BE, Birge RR (2002) Phototransduction by vertebrate ultraviolet visual pigments: protonation of the retinylidene Schiff base following photobleaching. Biochemistry 41:9842-9851.

Fahmy K, Zvyaga TA, Sakmar TP, Siebert F (1996) Spectroscopic evidence for altered chromophore-protein interactions in low-temperature photoproducts of the visual pigment responsible for congenital night blindness. Biochemistry 35:15065-15073.

Fasick JI, Applebury ML, Oprian DD (2002) Spectral tuning in the mammalian short-wavelength sensitive cone pigments. Biochemistry 41:6860-6865.

Feiler R, Harris WA, Kirschfeld K, Wehrhahn C, Zuker CS (1988) Targeted misexpression of a Drosophila opsin gene leads to altered visual function. Nature 333:737-741. 
Feiler R, Bjornson R, Kirschfeld K, Mismer D, Rubin GM, Smith DP, Socolich M, Zuker CS (1992) Ectopic expression of ultraviolet-rhodopsins in the blue photoreceptor cells of Drosophila: visual physiology and photochemistry of transgenic animals. J Neurosci 12:3862-3868.

Fisher CL, Pei GK (1997) Modification of a PCR-based site-directed mutagenesis method. Biotechniques 23:570-571.

Franceschini N (1979) Voltage clamp by light. Invest Opthalmol Suppl 6:5.

Franceschini N (1984) Chromatic organization and sexual dimorphism of the fly retinal mosaic. In: Photoreceptors (Borsellino A, Cervetto L, eds), pp 319-350. New York: Plenum.

Gärtner W (2000) Invertebrate visual pigements. In: Handbook of biological physics (Stavenga DG, De Grip WJ, Pugh EN, eds), pp 297-388. New York: Elsevier.

Gross AK, Xie G, Oprian DD (2003a) Slow binding of retinal to rhodopsin mutants G90D and T94D. Biochemistry 42:2002-2008.

Gross AK, Rao VR, Oprian DD (2003b) Characterization of rhodopsin congenital night blindness mutant T94I. Biochemistry 42:2009-2015.

Hashimoto S, Takeuchi H, Nakagawa M, Tsuda M (1996) Ultraviolet resonance Raman evidence for the absence of tyrosinate in octopus rhodopsin and the participation of Trp residues in the transition to acid metarhodopsin. FEBS Lett 398:239-242.

Hunt DM, Wilkie SE, Bowmaker JK, Poopalasundaram S (2001) Vision in the ultraviolet. Cell Mol Life Sci 58:1583-1598.

Jacobs GH (1981) Comparative color vision. New York: Academic.

Jager S, Lewis JW, Zvyaga TA, Szundi I, Sakmar TP, Kliger DS (1997) Timeresolved spectroscopy of the early photolysis intermediates of rhodopsin Schiff base counterion mutants. Biochemistry 36:1999-2009.

Janz JM, Farrens DL (2001) Engineering a functional blue-wavelengthshifted rhodopsin mutant. Biochemistry 40:7219-7227.

Jin S, Cornwall MC, Oprian DD (2003) Opsin activation as a cause of congenital night blindness. Nat Neurosci 6:731-735.

Karess R, Rubin G (1984) Analysis of P transposable element functions in Drosophila. Cell 38:135-146.

Kirschfeld K, Franceschini N, Minke B (1977) Evidence for a sensitising pigment in fly photoreceptors. Nature 269:386-390.

Lin SW, Kochendoerfer GG, Carroll KS, Wang D, Mathies RA, Sakmar TP (1998) Mechanisms of spectral tuning in blue cone visual pigments. Visible and raman spectroscopy of blue-shifted rhodopsin mutants. J Biol Chem 273:24583-24591.

Nakagawa M, Iwasa T, Kikkawa S, Tsuda M, Ebrey TG (1999) How vertebrate and invertebrate visual pigments differ in their mechanism of photoactivation. Proc Natl Acad Sci USA 96:6189-6192.

Nathans J (1990) Determinants of visual pigment absorbance: identification of the retinylidene Schiff's base counterion in bovine rhodopsin. Biochemistry 29:9746-9752.

O’Tousa JE, Baehr W, Martin RL, Hirsh J, Pak WL, Applebury ML (1985) The Drosophila ninaE gene encodes an opsin. Cell 40:839-850.

Palczewski K, Kumasaka T, Hori T, Behnke CA, Motoshima H, Fox BA, Le Trong I, Teller DC, Okada T, Stenkamp RE, Yamamoto M, Miyano M (2000) Crystal structure of rhodopsin: a G protein-coupled receptor. Science 289:739-745.

Pande C, Deng H, Rath P, Callender RH, Schwemer J (1987) Resonance raman spectroscopy of an ultraviolet-sensitive insect rhodopsin. Biochemistry 26:7426-7430.
Patton JS, Gomes XV, Geyer PK (1992) Position-independent germline transformation in Drosophila using a cuticle pigmentation gene as a selectable marker. Nucleic Acids Res 20:5859-5860.

Press WH, Teukolsky SA, Vetteringly WT, Flannery BR (1992) Numerical recipes in $\mathrm{C}$, the art of scientific computing, Ed 2. Cambridge, UK: Cambridge UP.

Rao VR, Cohen GB, Oprian DD (1994) Rhodopsin mutation G90D and a molecular mechanism for congenital night blindness. Nature 367:639-642.

Sakmar TP, Franke RR, Khorana HG (1989) Glutamic acid-113 serves as the retinylidene Schiff base counterion in bovine rhodopsin. Proc Natl Acad Sci USA 86:8309-8313.

Salcedo E, Huber A, Henrich S, Chadwell LV, Chou WH, Paulsen R, Britt SG (1999) Blue- and green-absorbing visual pigments of Drosophila: ectopic expression and physiological characterization of the R8 photoreceptor cell-specific Rh5 and Rh6 rhodopsins. J Neurosci 19:10716-10726.

Shi Y, Radlwimmer FB, Yokoyama S (2001) Molecular genetics and the evolution of ultraviolet vision in vertebrates. Proc Natl Acad Sci USA 98:11731-11736.

Sieving PA, Fowler ML, Bush RA, Machida S, Calvert PD, Green DG, Makino CL, McHenry CL (2001) Constitutive "light" adaptation in rods from G90D rhodopsin: a mechanism for human congenital nightblindness without rod cell loss. J Neurosci 21:5449-5460.

Stavenga DG, Smits RP, Hoenders BJ (1993) Simple exponential functions describing the absorbance bands of visual pigment spectra. Vision Res 33:1011-1017.

Tovee MJ (1995) Ultra-violet photoreceptors in the animal kingdom-their distribution and function. Trends Ecol Evol 10:455-460.

Townson SM, Chang BS, Salcedo E, Chadwell LV, Pierce NE, Britt SG (1998) Honeybee blue- and ultraviolet-sensitive opsins: cloning, heterologous expression in Drosophila, and physiological characterization. J Neurosci 18:2412-2422.

Weiner MP, Costa GL, Schoettlin W, Cline J, Mathur E, Bauer JC (1994) Site-directed mutagenesis of double-stranded DNA by the polymerase chain reaction. Gene 151:119-123.

Wilkie SE, Robinson PR, Cronin TW, Poopalasundaram S, Bowmaker JK, Hunt DM (2000) Spectral tuning of avian violet- and ultravioletsensitive visual pigments. Biochemistry 39:7895-7901.

Yan EC, Kazmi MA, Ganim Z, Hou JM, Pan D, Chang BS, Sakmar TP, Mathies RA (2003) Retinal counterion switch in the photoactivation of the G protein-coupled receptor rhodopsin. Proc Natl Acad Sci USA 100:9262-9267.

Yokoyama S, Radlwimmer FB, Blow NS (2000) Ultraviolet pigments in birds evolved from violet pigments by a single amino acid change. Proc Natl Acad Sci USA 97:7366-7371.

Zhukovsky EA, Oprian DD (1989) Effect of carboxylic acid side chains on the absorption maximum of visual pigments. Science 246:928-930.

Zuker CS, Cowman AF, Rubin GM (1985) Isolation and structure of a rhodopsin gene from D. melanogaster. Cell 40:851-858.

Zvyaga TA, Fahmy K, Siebert F, Sakmar TP (1996) Characterization of the mutant visual pigment responsible for congenital night blindness: a biochemical and Fourier-transform infrared spectroscopy study. Biochemistry 35:7536-7545. 